\title{
Development and Implementation of a Tenth-Order Hybrid Block Method for Solving Fifth-Order Boundary Value Problems
}

\author{
Higinio Ramos ${ }^{a}$ and Adelegan L. Momoh \\ ${ }^{a}$ Department of Applied Mathematics, Universidad de Salamanca \\ Plaza de la Merced, 37008 Salamanca, Spain \\ ${ }^{b}$ Department of Mathematical Sciences, Federal University of Technology \\ P.M.B. 704, Akure, Nigeria \\ E-mail(corresp.): almomoh@futa.edu.ng \\ E-mail: higra@usal.es
}

Received June 15, 2020; revised March 3, 2021; accepted March 3, 2021

Abstract. A hybrid convergent method of tenth-order is presented in this work for directly solving fifth-order boundary value problems in ordinary differential equations. A unique direct block approach is obtained by combining multiple Finite Difference Formulas which are derived via the collocation technique. The proposed method is fully analyzed and the existence and uniqueness of the discrete solution is established. Different numerical examples are considered and the results are compared with those provided by existing works in the literature. The comparison shows the good performance of the present method over some cited works in the literature, confirming the competitiveness and superiority of the new numerical integrator.

Keywords: block method, fifth-order boundary value problem, convergence analysis, existence and uniqueness of solution, ordinary differential equations.

AMS Subject Classification: 65L05; 65L06; 65L10.

\section{Introduction}

This paper considers the direct numerical solution of fifth-order BVPs of the form

$$
\left\{\begin{array}{l}
y^{(5)}=f\left(x, y, y^{\prime}, y^{\prime \prime} y^{\prime \prime \prime}, y^{(4)}\right), \quad x \in[a, b] \\
y(a)=\alpha_{0}, \quad y(b)=\beta_{0}, \\
y^{\prime}(a)=\alpha_{1}, \quad y^{\prime}(b)=\beta_{1}, \\
y^{\prime \prime}(a)=\alpha_{2} .
\end{array}\right.
$$

Copyright (C) 2021 The Author(s). Published by Vilnius Gediminas Technical University

This is an Open Access article distributed under the terms of the Creative Commons Attribution License (http://creativecommons.org/licenses/by/4.0/), which permits unrestricted use, distribution, and reproduction in any medium, provided the original author and source are credited. 
where $\alpha_{0}, \alpha_{1}, \alpha_{2}, \beta_{0}$ and $\beta_{1}$ are real constants, and $f$ is assumed to be a continuous function on a prescribed domain of interest. Problems of this nature usually arise in the mathematical modelling of viscoelastic flows, induction motor and different aspects of mathematical, physical and engineering sciences (see, $[5,16,18])$. The theorems that provide the conditions for existence and uniqueness of solutions of the boundary value problems of type (1.1) are extensively discussed in [1].

As it is usually difficult or impossible to obtain exact solutions for this type of problem, the approximation is made by applying semi-analytical methods such as Adomian decomposition, spline method, variational iterative method, septic spline method, Legendre-homotopy method and others (see, $[2,3,5,13$, 14, 15, 16, 18, 19]). Caglar et al. [3] presented a method for solving fifth-order boundary value problems where they adopted an approximation by a sixthdegree B-spline function and exhibited a first order convergence. Wazwaz [18] applied Adomian and modified Adomian decomposition methods to deal with it, while Siddiqi and Akram presented a sixtic spline method with second order convergence. Zhang [19] proposed a variational iteration method as an improvement over Adomian and six-degree B-spline methods in [3] and [18].

In this work, a tenth-order block method for directly solving a fifth-order boundary value problem which is assumed to have a unique solution within the integration interval is presented. To develop the method, the solution is sought on an interval of the form $\left[x_{n}, x_{n+2}\right]$, with inclusion of two intermediate points. To further improve the order and the stability of the method, we have considered sixth derivative terms. This resulted into a method that is of uniform tenth theoretical order which is capable of handling directly the solution of equations of the type in (1.1).

The rest of this paper is arranged as follows: in Section 2, the development of the proposed method is presented. The characteristics of the method are discussed in Section 3, while in Sections 4 and 5, the implementation details and some numerical examples are reported. Finally, some conclusions are presented in Section 6.

\section{Development of the method}

The usual practice in many numerical methods for obtaining an approximate solution for the equation of type (1.1) is to assume that its solution $y(x)$ can be approximated by a polynomial $p(x)$. The discrete numerical approximation of the problem is considered on an interval $[a, b]$ with a set of grid points $\left\{a=x_{0}<x_{1}<\cdots<x_{N}=b\right\}$, and a constant step size $h=x_{i+1}-x_{i}, \quad i=$ $0,1, \ldots, N-1$. To derive the method, let us consider a generic two-block subinterval $\left[x_{n}, x_{n+2}\right]$ and assume that the theoretical solution to (1.1) is approximated here by a polynomial of the form:

$$
y(x) \approx p(x)=\sum_{r=0}^{14} a_{r} x^{r},
$$


where $a_{r}, r=0,1, \ldots, 14$, are parameters to be determined. These parameters are uniquely obtained by imposing the following conditions:

$$
\begin{aligned}
& p^{(i)}\left(x_{n}\right)=y_{n}^{(i)}, \quad i=0,1,2,3,4, \\
& p^{(5)}\left(x_{n+\frac{\bar{i}}{2}}\right)=f_{n+\frac{\bar{i}}{2}}, \quad \bar{i}=0,1,2,3,4, \\
& p^{(6)}\left(x_{n+\frac{\bar{i}}{2}}\right)=g_{n+\frac{\bar{i}}{2}}, \quad \bar{i}=0,1,2,3,4,
\end{aligned}
$$

where $f_{j}, g_{j}$ are respectively the approximations at the corresponding grid points of $f\left(x, y, y^{\prime}, y^{\prime \prime}, y^{\prime \prime \prime}, y^{(4)}\right)$ and $g\left(x, y, y^{\prime}, y^{\prime \prime}, y^{\prime \prime \prime}, y^{(4)}\right)=\frac{d f\left(x, y, y^{\prime}, y^{\prime \prime}, y^{\prime \prime \prime}, y^{(4)}\right)}{d x}$. The successive derivatives of (2.1) are obtained to be

$$
\begin{aligned}
& y^{\prime}(x) \approx p^{\prime}(x)=\sum_{r=1}^{14} D_{1, r} a_{r} x^{r-1}, \quad y^{\prime \prime}(x) \approx p^{\prime \prime}(x)=\sum_{r=2}^{14} D_{2, r} a_{r} x^{r-2}, \\
& y^{\prime \prime \prime}(x) \approx p^{\prime \prime \prime}(x)=\sum_{r=3}^{14} D_{3, r} a_{r} x^{r-3}, \quad y^{(4)}(x) \approx p^{(4)}(x)=\sum_{r=4}^{14} D_{4, r} a_{r} x^{r-4}, \\
& y^{(5)}(x) \approx p^{(5)}(x)=\sum_{r=5}^{14} D_{5, r} a_{r} x^{r-5}, \quad y^{(6)}(x) \approx p^{(6)}(x)=\sum_{r=6}^{14} D_{6, r} a_{r} x^{r-6},
\end{aligned}
$$

where $D_{j, r}=\prod_{s=0}^{j-1}(r-s), j=1,2, \ldots, 6$. We impose the conditions given in (2.2)-(2.4) using the approximations in (2.1) and (2.5).This leads to a system of linear equations that can be expressed in matrix form as

$$
W A=F,
$$

where

$$
\begin{aligned}
& W=\left(\begin{array}{ccccccccc}
1 & x_{n} & x_{n}^{2} & x_{n}^{3} & x_{n}^{4} & x_{n}^{5} & x_{n}^{6} & \ldots & x_{n}^{k} \\
0 & 1 & 2 x_{n} & 3 x_{n}^{2} & 4 x_{n}^{3} & 5 x_{n}^{4} & 6 x_{n}^{5} & \ldots & D_{1, k} x_{n}^{k-1} \\
0 & 0 & 2 & 6 x_{n} & 12 x_{n}^{2} & 20 x_{n}^{3} & 30 x_{n}^{4} & \ldots & D_{2, k} x_{n}^{k-2} \\
0 & 0 & 0 & 6 & 24 x_{n} & 60 x_{n}^{2} & 120 x_{n}^{3} & \ldots & D_{3, k} x_{n}^{k-3} \\
0 & 0 & 0 & 0 & 24 & 120 x_{n} & 360 x_{n}^{2} & \ldots & D_{4, k} x_{n}^{k-4} \\
0 & 0 & 0 & 0 & 0 & 120 & 720 x_{n} & \ldots & D_{5, k} x_{n}^{k-5} \\
\vdots & \vdots & \vdots & \vdots & \vdots & \vdots & \vdots & \vdots & \vdots \\
0 & 0 & 0 & 0 & 0 & 120 & 720 x_{n+2} & \ldots & D_{5, k} x_{n+5}^{k-5} \\
0 & 0 & 0 & 0 & 0 & 0 & 720 & \ldots & D_{6, k} x_{n}^{k-6} \\
\vdots & \vdots & \vdots & \vdots & \vdots & \vdots & \vdots & \vdots & \vdots \\
0 & 0 & 0 & 0 & 0 & 0 & 720 & \ldots & D_{6, k} x_{n+2}^{k-6}
\end{array}\right), \\
& A=\left(a_{0}, a_{1}, a_{2}, a_{3}, a_{4}, a_{5}, \ldots, a_{k}\right)^{T} \text {, } \\
& F=\left(y_{n}, y_{n}^{\prime}, y_{n}^{\prime \prime}, y_{n}^{\prime \prime \prime}, y_{n}^{(4)}, f_{n}, f_{n+\frac{1}{2}}, \ldots, f_{n+2}, g_{n}, g_{n+\frac{1}{2}}, \ldots, g_{n+2}\right)^{T} \text {, }
\end{aligned}
$$

being $k=14$. Now we proceed by solving (2.6) to get the parameters $a_{r}$ 's using Gaussian elimination with the aid of the CAS Mathematica. These values are 
then substituted into (2.1) and after some simplification yields a continuous representation of the approximating polynomial in the form:

$$
p(x)=\sum_{\bar{i}=0}^{4} \alpha_{\bar{i}}(x) y_{n}^{(\bar{i})} h^{\bar{i}}+h^{5} \sum_{\bar{i}=0}^{4} \beta_{\bar{i}}(x) f_{n+\frac{\bar{i}}{2}}+h^{6} \sum_{\bar{i}=0}^{4} \gamma_{\bar{i}}(x) g_{n+\frac{\bar{i}}{2}},
$$

where $\alpha_{\bar{i}}(x), \beta_{\bar{i}}(x)$ and $\gamma_{\bar{i}}(x), \bar{i}=0(1) 4$, are continuous coefficients and $h$ is the fixed step-size. We do not present here these coefficients as they are cumbersome expressions, and can be easily obtained with a CAS.

\subsection{Main formulas}

We obtained the main members of the tenth-order block method by substituting the values of $\alpha_{\bar{i}}(x), \beta_{\bar{i}}(x), \gamma_{\bar{i}}(x), i=0(1) 4$ in (2.7) and evaluating $p\left(x_{n}+\frac{\bar{i} h}{2}\right)$ to get approximations for $y\left(x_{n}+\frac{\bar{i} h}{2}\right), \bar{i}=1,2,3$, 4. The resulting formulas are as presented in Table 1 alongside the additional formulas.

\subsection{Additional formulas}

In order to obtain the necessary additional formulas to form the block method, we consider the first, second, third and fourth derivatives of $p(x)$ in $(2.7)$ and then evaluate $p^{(i)}\left(x_{n}+\frac{\bar{i} h}{2}\right)$ to get approximations for $y^{(i)}\left(x_{n}+\frac{\bar{i} h}{2}\right), \bar{i}=1,2,3,4$. A total of sixteen additional formulas are obtained which shall be combined as a block for numerically solving BVPs of the type in (1.1). All the formulas are schematically presented in Table 1 below.

\section{Characteristics of the method}

\subsection{Local truncation error and order}

Suppose $y(x)$ is a sufficiently differentiable function which is the true solution of (1.1). The linear difference operators associated with the formulas in Table 1 are given as

$$
\begin{aligned}
& \mathbb{L}_{\frac{i}{2}}[y(x) ; h] \equiv y\left(x+\frac{i}{2} h\right)-\left[\sum_{m=0}^{4} \alpha_{m}\left(x+\frac{i}{2} h\right) y^{(m)}(x) h^{m}\right. \\
& \left.\quad+h^{5} \sum_{m=0}^{4} \beta_{m}\left(x+\frac{i}{2} h\right) y^{(5)}\left(x+\frac{i}{2} h\right)+h^{6} \sum_{m=0}^{4} \gamma_{m}\left(x+\frac{i}{2} h\right) y^{(6)}\left(x+\frac{i}{2} h\right)\right],
\end{aligned}
$$

where $i=1(1) 4$. The Taylor's series expansion of (3.1) around $x$ yields the truncation errors of the form 


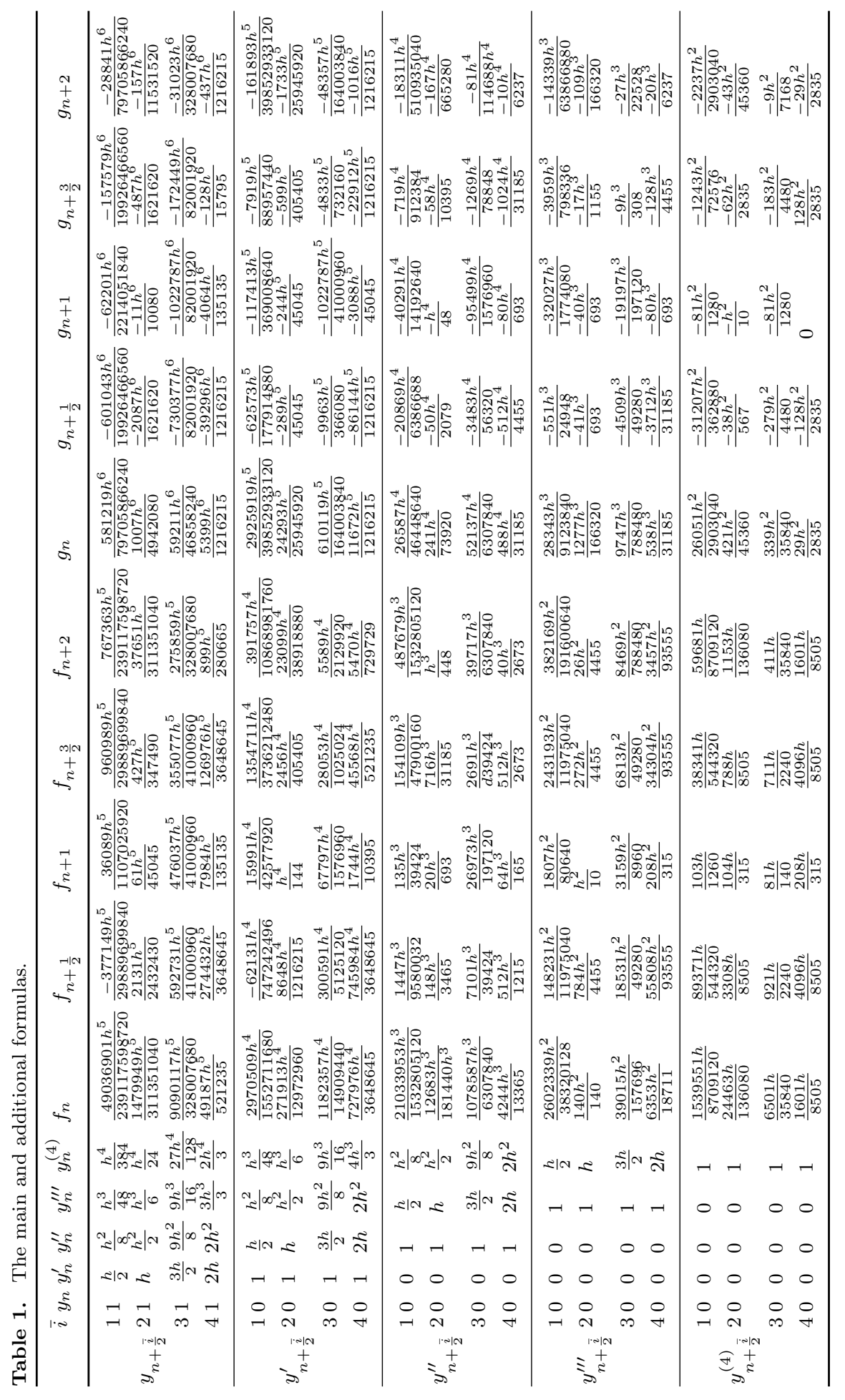




$$
\mathbb{L}_{\frac{i}{2}}[y(x) ; h]=\check{\mathrm{c}}_{0} y(x)+\check{\mathrm{c}}_{1} h y^{\prime}(x)+\check{\mathrm{c}}_{2} h^{2} y^{\prime \prime}(x)+\ldots+\check{\mathrm{c}}_{j} h^{j} y^{(j)}(x)+O\left(h^{j+1}\right),
$$

where $\check{\mathrm{c}}_{j}$ are constants. If the first $p+5$ constants are equal to zero, which means that $\check{\mathrm{c}}_{0}=\check{\mathrm{c}}_{1}=\check{\mathrm{c}}_{2}=\ldots=\check{\mathrm{c}}_{p+4}=0$, and $\check{\mathrm{c}}_{p+5} \neq 0$, this implies that

$$
\mathbb{L}_{\frac{i}{2}}[y(x) ; h]=\check{\mathrm{c}}_{p+5} h^{p+5} y^{p+5}(x)+O\left(h^{p+6}\right),
$$

where $p$ and $\check{c}_{p+5}$ are respectively known as the order and local principal error constant of the corresponding formula (see $[4,6,9,10]$ ). The local truncation errors and the principal error constants of the main formulas are obtained to be:

$$
\begin{aligned}
\mathbb{L}_{\frac{1}{2}}[y(x) ; h] & =\frac{107831}{257099242143744000} y^{(15)}(x) h^{15}+O\left(h^{16}\right), \\
\mathbb{L}_{1}[y(x) ; h] & =\frac{11}{713276928000} y^{(15)}(x) h^{15}+O\left(h^{16}\right), \\
\mathbb{L}_{\frac{3}{2}}[y(x) ; h] & =\frac{1773}{16793993216000} y^{(15)}(x) h^{15}+O\left(h^{16}\right), \\
\mathbb{L}_{2}[y(x) ; h] & =\frac{193}{490377888000} y^{(15)}(x) h^{15}+O\left(h^{16}\right) .
\end{aligned}
$$

For the additional formulas, the local truncation errors can be obtained in a similar manner. It results that the formulas are of theoretical order $p=10$.

\subsection{Convergence analysis}

In this section, the convergence analysis of the proposed method, which will be named in short BDM5, is addressed.

Definition 1. (see [11]) Let $y(x)$ be the theoretical solution of (1.1) and $\left\{y_{j}\right\}_{j \in J}$ be the approximate solution at the grid points obtained by adopting BDM5, that is $y_{j} \simeq y\left(x_{j}\right)$. BDM5 is to be of $p^{t h}-$ theoretical order of convergence if for $h$ sufficiently small, there exists a constant $k$ independent of $h$ such that

$$
\max _{j \in J}\left\|y\left(x_{j}\right)-y_{j}\right\| \leq k h^{p}
$$

This implies that

$$
\max _{0 \leq j \leq N}\left\|y\left(x_{j}\right)-y_{j}\right\| \rightarrow 0 \text { as } h \rightarrow 0 .
$$

The convergence of the proposed method will be established by expressing the main and additional formulas in matrix form adopting the following notations. Let $\bar{M}$ be a $10 N \times 10 N$ matrix defined as

$$
\bar{M}=\left(\begin{array}{lllll}
m_{11} & m_{12} & m_{13} & m_{14} & m_{15} \\
m_{21} & m_{22} & m_{23} & m_{24} & m_{25} \\
m_{31} & m_{32} & m_{33} & m_{34} & m_{35} \\
m_{41} & m_{42} & m_{43} & m_{44} & m_{45} \\
m_{51} & m_{52} & m_{53} & m_{54} & m_{55}
\end{array}\right),
$$

where 


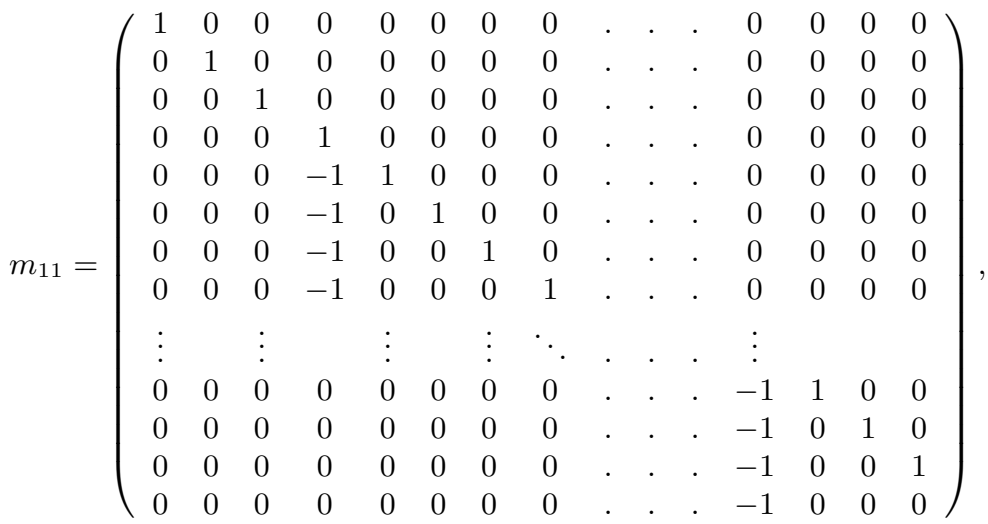

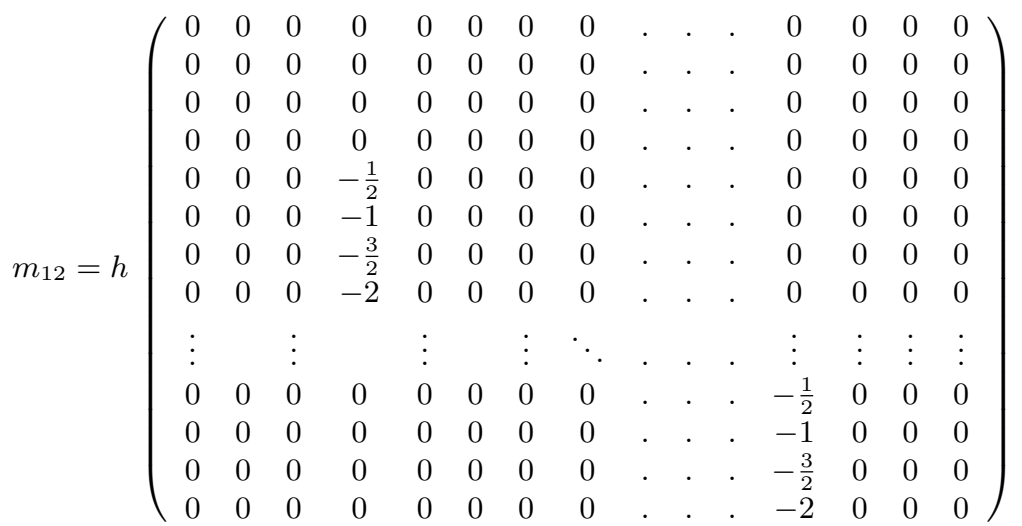

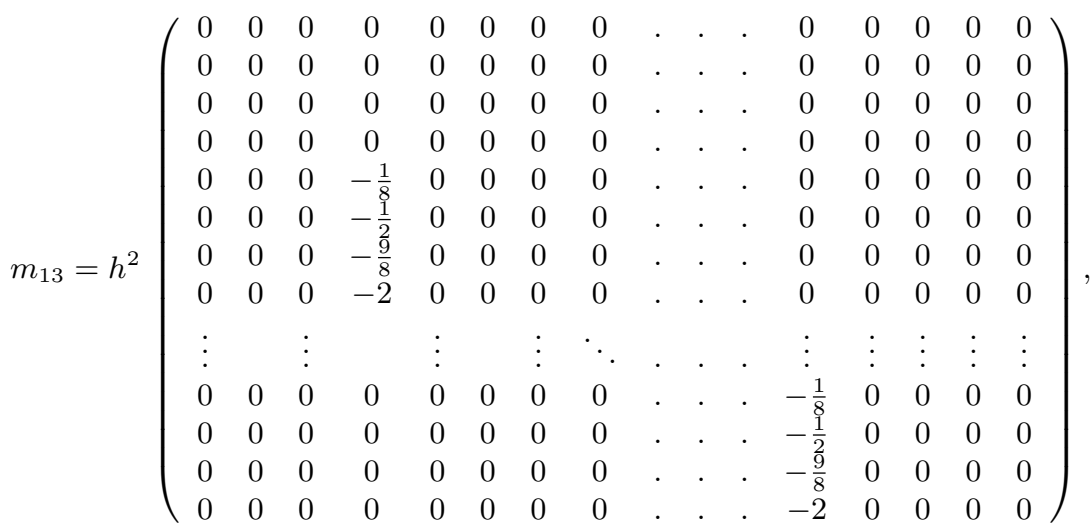




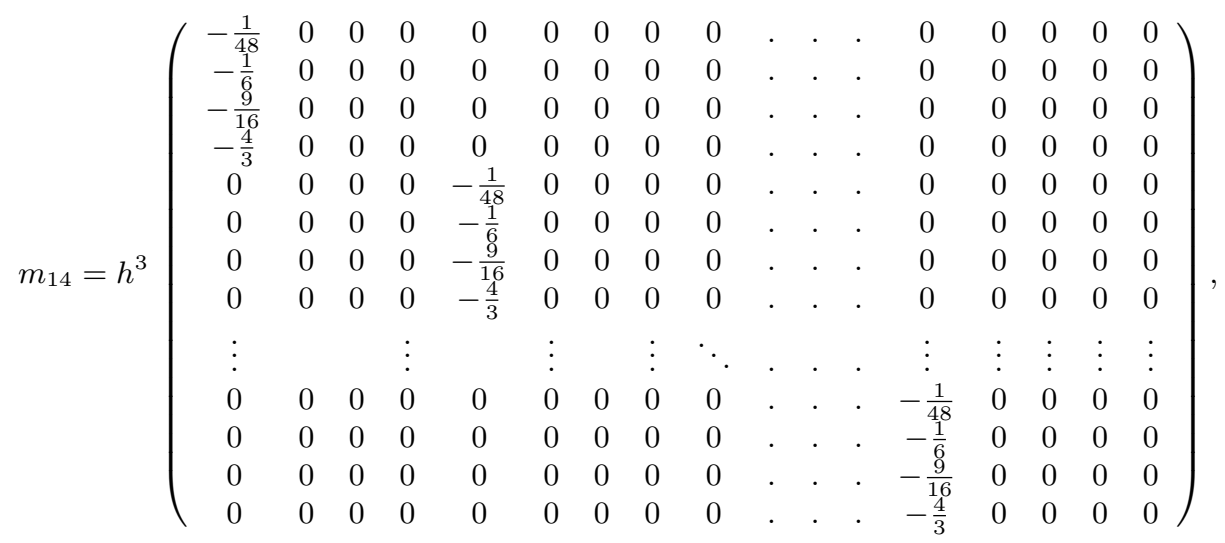

$$
m_{15}=h^{4}\left(\begin{array}{ccccccccccccccc}
\frac{-1}{384} & 0 & 0 & 0 & 0 & 0 & 0 & 0 & 0 & \ldots & 0 & 0 & 0 & 0 & 0 \\
\frac{-1}{24} & 0 & 0 & 0 & 0 & 0 & 0 & 0 & 0 & \ldots & 0 & 0 & 0 & 0 & 0 \\
\frac{-27}{128} & 0 & 0 & 0 & 0 & 0 & 0 & 0 & 0 & \ldots & 0 & 0 & 0 & 0 & 0 \\
\frac{-2}{3} & 0 & 0 & 0 & 0 & 0 & 0 & 0 & 0 & \ldots & 0 & 0 & 0 & 0 & 0 \\
0 & 0 & 0 & 0 & \frac{-1}{384} & 0 & 0 & 0 & 0 & \ldots & 0 & 0 & 0 & 0 & 0 \\
0 & 0 & 0 & 0 & \frac{-1}{24} & 0 & 0 & 0 & 0 & \ldots & 0 & 0 & 0 & 0 & 0 \\
0 & 0 & 0 & 0 & \frac{-27}{128} & 0 & 0 & 0 & 0 & \ldots & 0 & 0 & 0 & 0 & 0 \\
0 & 0 & 0 & 0 & \frac{-2}{3} & 0 & 0 & 0 & 0 & \ldots & 0 & 0 & 0 & 0 & 0 \\
\vdots & & & \vdots & & \vdots & & \vdots & \ddots & \ldots & \vdots & \vdots & \vdots & \vdots & \vdots \\
0 & 0 & 0 & 0 & 0 & 0 & 0 & 0 & 0 & \ldots & \frac{-1}{384} & 0 & 0 & 0 & 0 \\
0 & 0 & 0 & 0 & 0 & 0 & 0 & 0 & 0 & \ldots & \frac{-1}{24} & 0 & 0 & 0 & 0 \\
0 & 0 & 0 & 0 & 0 & 0 & 0 & 0 & 0 & \ldots & \frac{-37}{128} & 0 & 0 & 0 & 0 \\
0 & 0 & 0 & 0 & 0 & 0 & 0 & 0 & 0 & \ldots & \frac{-2}{3} & 0 & 0 & 0 & 0
\end{array}\right),
$$

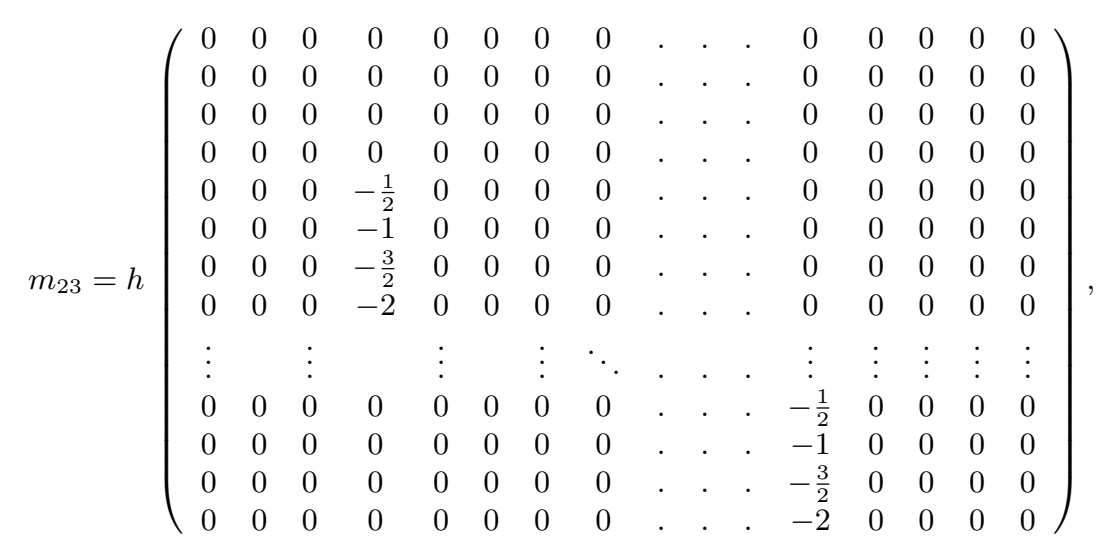




$$
m_{24}=h^{2}\left(\begin{array}{ccccccccccccccc}
-\frac{1}{8} & 0 & 0 & 0 & 0 & 0 & 0 & 0 & 0 & \ldots & 0 & 0 & 0 & 0 & 0 \\
-\frac{1}{2} & 0 & 0 & 0 & 0 & 0 & 0 & 0 & 0 & \ldots & 0 & 0 & 0 & 0 & 0 \\
-\frac{9}{8} & 0 & 0 & 0 & 0 & 0 & 0 & 0 & 0 & \ldots & 0 & 0 & 0 & 0 & 0 \\
-2 & 0 & 0 & 0 & 0 & 0 & 0 & 0 & 0 & \ldots & 0 & 0 & 0 & 0 & 0 \\
0 & 0 & 0 & 0 & -\frac{1}{8} & 0 & 0 & 0 & 0 & \ldots & 0 & 0 & 0 & 0 & 0 \\
0 & 0 & 0 & 0 & -\frac{1}{2} & 0 & 0 & 0 & 0 & \ldots & 0 & 0 & 0 & 0 & 0 \\
0 & 0 & 0 & 0 & -\frac{9}{8} & 0 & 0 & 0 & 0 & \ldots & 0 & 0 & 0 & 0 & 0 \\
0 & 0 & 0 & 0 & -2 & 0 & 0 & 0 & 0 & \ldots & 0 & 0 & 0 & 0 & 0 \\
\vdots & & & \vdots & & \vdots & & \vdots & \ddots & \ldots & \vdots & \vdots & \vdots & \vdots & \vdots \\
0 & 0 & 0 & 0 & 0 & 0 & 0 & 0 & 0 & \ldots & -\frac{1}{8} & 0 & 0 & 0 & 0 \\
0 & 0 & 0 & 0 & 0 & 0 & 0 & 0 & 0 & \ldots & -\frac{1}{2} & 0 & 0 & 0 & 0 \\
0 & 0 & 0 & 0 & 0 & 0 & 0 & 0 & 0 & \ldots & -\frac{9}{8} & 0 & 0 & 0 & 0 \\
0 & 0 & 0 & 0 & 0 & 0 & 0 & 0 & 0 & \ldots & -2 & 0 & 0 & 0 & 0
\end{array}\right),
$$$$
m_{33}=\left(\begin{array}{cccccccccccccccc}
1 & 0 & 0 & 0 & 0 & 0 & 0 & 0 & . & . & . & 0 & 0 & 0 & 0 & 0 \\
0 & 1 & 0 & 0 & 0 & 0 & 0 & 0 & . & . & . & 0 & 0 & 0 & 0 & 0 \\
0 & 0 & 1 & 0 & 0 & 0 & 0 & 0 & . & . & . & 0 & 0 & 0 & 0 & 0 \\
0 & 0 & 0 & 1 & 0 & 0 & 0 & 0 & . & . & . & 0 & 0 & 0 & 0 & 0 \\
0 & 0 & 0 & -1 & 1 & 0 & 0 & 0 & . & . & . & 0 & 0 & 0 & 0 & 0 \\
0 & 0 & 0 & -1 & 0 & 1 & 0 & 0 & . & . & . & 0 & 0 & 0 & 0 & 0 \\
0 & 0 & 0 & -1 & 0 & 0 & 1 & 0 & . & . & . & 0 & 0 & 0 & 0 & 0 \\
0 & 0 & 0 & -1 & 0 & 0 & 0 & 1 & . & . & . & 0 & 0 & 0 & 0 & 0 \\
\vdots & & \vdots & & \vdots & & \vdots & . & & & . & . & & & & \\
0 & 0 & 0 & 0 & 0 & 0 & 0 & 0 & . & . & . & -1 & . & . & . & . \\
0 & 0 & 0 & 0 & 0 & 0 & 0 & 0 & . & . & . & -1 & 0 & 1 & 0 & 0 \\
0 & 0 & 0 & 0 & 0 & 0 & 0 & 0 & . & . & . & -1 & 0 & 0 & 1 & 0 \\
0 & 0 & 0 & 0 & 0 & 0 & 0 & 0 & . & . & . & -1 & 0 & 0 & 0 & 1
\end{array}\right),
$$

$$
m_{34}=h\left(\begin{array}{ccccccccccccccc}
-\frac{1}{2} & 0 & 0 & 0 & 0 & 0 & 0 & 0 & 0 & \ldots & 0 & 0 & 0 & 0 & 0 \\
-1 & 0 & 0 & 0 & 0 & 0 & 0 & 0 & 0 & \ldots & 0 & 0 & 0 & 0 & 0 \\
-\frac{3}{2} & 0 & 0 & 0 & 0 & 0 & 0 & 0 & 0 & \ldots & 0 & 0 & 0 & 0 & 0 \\
-2 & 0 & 0 & 0 & 0 & 0 & 0 & 0 & 0 & \ldots & 0 & 0 & 0 & 0 & 0 \\
0 & 0 & 0 & 0 & -\frac{1}{2} & 0 & 0 & 0 & 0 & \ldots & 0 & 0 & 0 & 0 & 0 \\
0 & 0 & 0 & 0 & -1 & 0 & 0 & 0 & 0 & \ldots & 0 & 0 & 0 & 0 & 0 \\
0 & 0 & 0 & 0 & -\frac{3}{2} & 0 & 0 & 0 & 0 & \ldots & 0 & 0 & 0 & 0 & 0 \\
0 & 0 & 0 & 0 & -2 & 0 & 0 & 0 & 0 & \ldots & 0 & 0 & 0 & 0 & 0 \\
\vdots & & & \vdots & & \vdots & & \vdots & \ddots & \ldots & \vdots & \vdots & \vdots & \vdots & \vdots \\
0 & 0 & 0 & 0 & 0 & 0 & 0 & 0 & 0 & \ldots & -\frac{1}{2} & 0 & 0 & 0 & 0 \\
0 & 0 & 0 & 0 & 0 & 0 & 0 & 0 & 0 & \ldots & -1 & 0 & 0 & 0 & 0 \\
0 & 0 & 0 & 0 & 0 & 0 & 0 & 0 & 0 & \ldots & -\frac{3}{2} & 0 & 0 & 0 & 0 \\
0 & 0 & 0 & 0 & 0 & 0 & 0 & 0 & 0 & \ldots & -2 & 0 & 0 & 0 & 0
\end{array}\right)
$$




$$
m_{44}=\left(\begin{array}{ccccccccccccccc}
-1 & 1 & 0 & 0 & 0 & 0 & 0 & 0 & 0 & \ldots & 0 & 0 & 0 & 0 & 0 \\
-1 & 0 & 1 & 0 & 0 & 0 & 0 & 0 & 0 & \ldots & 0 & 0 & 0 & 0 & 0 \\
-1 & 0 & 0 & 1 & 0 & 0 & 0 & 0 & 0 & \ldots & 0 & 0 & 0 & 0 & 0 \\
-1 & 0 & 0 & 0 & 1 & 0 & 0 & 0 & 0 & \ldots & 0 & 0 & 0 & 0 & 0 \\
0 & 0 & 0 & 0 & -1 & 1 & 0 & 0 & 0 & \ldots & 0 & 0 & 0 & 0 & 0 \\
0 & 0 & 0 & 0 & -1 & 0 & 1 & 0 & 0 & \ldots & 0 & 0 & 0 & 0 & 0 \\
0 & 0 & 0 & 0 & -1 & 0 & 0 & 1 & 0 & \ldots & 0 & 0 & 0 & 0 & 0 \\
0 & 0 & 0 & 0 & -1 & 0 & 0 & 0 & 1 & \ldots & 0 & 0 & 0 & 0 & 0 \\
\vdots & & & \vdots & & \vdots & & \vdots & \ddots & \ldots & \vdots & . & . & . & . \\
0 & 0 & 0 & 0 & 0 & 0 & 0 & 0 & 0 & \ldots & -1 & 1 & 0 & 0 & 0 \\
0 & 0 & 0 & 0 & 0 & 0 & 0 & 0 & 0 & \ldots & -1 & 0 & 1 & 0 & 0 \\
0 & 0 & 0 & 0 & 0 & 0 & 0 & 0 & 0 & \ldots & -1 & 0 & 0 & 1 & 0 \\
0 & 0 & 0 & 0 & 0 & 0 & 0 & 0 & 0 & \ldots & -1 & 0 & 0 & 0 & 1
\end{array}\right),
$$

where $m_{11}=m_{22}, m_{14}=m_{25}, m_{24}=m_{35}, m_{34}=m_{45}, m_{44}=m_{55}$. This reduces the matrix $\bar{M}$ to an upper triangular matrix with $m_{i j}=0, i>j$. We note that the above matrices are related with the coefficients of the formulas given in Table 1 . Similarly, let $\bar{N}$ be defined as a $10 N \times(4 N+2)$ matrix of the form

$$
\bar{N}=\left(\begin{array}{lllll}
n_{11} & n_{21} & n_{31} & n_{41} & n_{51} \\
n_{12} & n_{22} & n_{32} & n_{42} & n_{52}
\end{array}\right)^{T}
$$

where the entries are obtained from Table 1 similarly as was done for $M$. It should be noted that $n_{i 1}$ and $n_{i 2}, i=1,2, \ldots, 5$ are $2 N \times(2 N+1)$ non-zero matrices. The entries of the submatrices $m_{i j}$ and $n_{i j}$ are the coefficients of the formulas in Table 1. Let define the following vectors corresponding to the exact values $y(x)$ and its derivatives

$$
\begin{aligned}
\bar{Y}= & \left(y\left(x_{\frac{1}{2}}\right), y\left(x_{1}\right), \ldots, y\left(x_{N-\frac{1}{2}}\right), y^{\prime}\left(x_{\frac{1}{2}}\right), \ldots, y^{\prime}\left(x_{N-\frac{1}{2}}\right), y^{\prime \prime}\left(x_{\frac{1}{2}}\right), \ldots, y^{\prime \prime}\left(x_{N}\right),\right. \\
& \left.y^{\prime \prime \prime}\left(x_{0}\right), y^{\prime \prime \prime}\left(x_{\frac{1}{2}}\right), \ldots, y^{\prime \prime \prime}\left(x_{N}\right), y^{i v}\left(x_{0}\right), y^{i v}\left(x_{\frac{1}{2}}\right), \ldots, y^{i v}\left(x_{N}\right)\right)^{T}, \\
\bar{F}= & \left(f\left(x_{0}, y\left(x_{0}\right), y^{\prime}\left(x_{0}\right), y^{\prime \prime}\left(x_{0}\right), y^{\prime \prime \prime}\left(x_{0}\right), y^{i v}\left(x_{0}\right)\right), f\left(x_{\frac{1}{2}}, y\left(x_{\frac{1}{2}}\right), y^{\prime}\left(x_{\frac{1}{2}}\right), y^{\prime \prime}\left(x_{\frac{1}{2}}\right),\right.\right. \\
& \left.y^{\prime \prime \prime}\left(x_{\frac{1}{2}}\right), y^{i v}\left(x_{\frac{1}{2}}\right)\right), \ldots, f\left(x_{N}, y\left(x_{N}\right), y^{\prime}\left(x_{N}\right), y^{\prime \prime}\left(x_{N}\right), y^{\prime \prime \prime}\left(x_{N}\right), y^{i v}\left(x_{N}\right)\right), \\
& g\left(x_{0}, y\left(x_{0}\right), y^{\prime}\left(x_{0}\right), y^{\prime \prime}\left(x_{0}\right), y^{\prime \prime \prime}\left(x_{0}\right), y^{i v}\left(x_{0}\right)\right), g\left(x_{\frac{1}{2}}, y\left(x_{\frac{1}{2}}\right), y^{\prime}\left(x_{\frac{1}{2}}\right), y^{\prime \prime}\left(x_{\frac{1}{2}}\right),\right. \\
& \left.\left.y^{\prime \prime \prime}\left(x_{\frac{1}{2}}\right), y^{i v}\left(x_{\frac{1}{2}}\right)\right), \ldots, g\left(x_{N}, y\left(x_{N}\right), y^{\prime}\left(x_{N}\right), y^{\prime \prime}\left(x_{N}\right), y^{\prime \prime \prime}\left(x_{N}\right), y^{i v}\left(x_{N}\right)\right)\right)^{T},
\end{aligned}
$$

where $\bar{Y}$ is a $10 N$-vector and $\bar{F}$ is a $(4 N+2)$-vector. Putting the above notation into account, it is possible to write

$$
\bar{M}_{10 N \times 10 N} \bar{Y}_{10 N}+h^{5} \bar{N}_{10 N \times(4 N+2)} \bar{F}_{4 N+2}+\mathbf{C}_{10 N}=\mathbb{L}(h)_{10 N},
$$

which represents the exact form of the system that approximates (1.1). $\mathbf{C}_{10 \mathrm{~N}}$ 
is a vector which contains the known values, defined as

$$
\begin{aligned}
\mathbf{C}_{10 N}=( & -y_{0}-\frac{h}{2} y_{0}^{\prime}-\frac{h^{2}}{8} y_{0}^{\prime \prime},-y_{0}-h y_{0}^{\prime}-\frac{h^{2}}{2} y_{0}^{\prime \prime},-y_{0}-\frac{3 h}{2} y_{0}^{\prime}-\frac{9 h^{2}}{8} y_{0}^{\prime \prime}, \\
& -y_{0}-2 h y_{0}^{\prime}-2 h y_{0}^{\prime \prime}, 0, \ldots, 0,-y_{0}^{\prime}-\frac{h}{2} y_{0}^{\prime \prime},-y_{0}^{\prime}-h y_{0}^{\prime \prime},-y_{0}^{\prime}-\frac{3 h}{2} y_{0}^{\prime \prime}, \\
& -y_{0}^{\prime}-2 h y_{0}^{\prime \prime}, 0, \ldots, 0,-y_{0}^{\prime \prime}-\frac{h}{2} y_{0}^{\prime \prime \prime},-y_{0}^{\prime \prime}-h y_{0}^{\prime \prime \prime},-y_{0}^{\prime \prime}-\frac{3 h}{2} y_{0}^{\prime \prime \prime}, \\
& \left.-y_{0}^{\prime \prime}-2 h y_{0}^{\prime \prime \prime}, 0, \ldots, 0\right)^{T},
\end{aligned}
$$

while $\mathbb{L}(h)_{10 N}$ represents the local truncation errors of the formulas in Table 1 which are given as

$$
\begin{aligned}
& \mathbb{L}(h)_{10 N}=\left(\frac{107831}{257099242143744000} y^{(15)}\left(x_{0}\right) h^{15}+O\left(h^{16}\right), \frac{11}{713276928000} h^{15}\right. \\
& \times y^{(15)}\left(x_{0}\right)+O\left(h^{16}\right), \frac{1773}{16793993216000} y^{(15)}\left(x_{0}\right) h^{15}+O\left(h^{16}\right), \\
& \frac{193}{490377888000} y^{(15)}\left(x_{0}\right) h^{15}+O\left(h^{16}\right), \frac{107831}{257099242143744000} y^{(15)}\left(x_{1}\right) h^{15}+O\left(h^{16}\right), \\
& \frac{11}{713276928000} y^{(15)}\left(x_{1}\right) h^{15}+O\left(h^{16}\right), \frac{1773}{16793993216000} y^{(15)}\left(x_{1}\right) h^{15}+O\left(h^{16}\right), \\
& \frac{193}{490377888000} y^{(15)}\left(x_{1}\right) h^{15}+O\left(h^{16}\right), \ldots, \frac{193}{490377888000} y^{(15)}\left(x_{N-2}\right) h^{15}+O\left(h^{16}\right), \\
& \frac{601043}{128549621071872000} y^{(15)}\left(x_{0}\right) h^{15}+O\left(h^{16}\right), \ldots, \frac{437}{490377888000} y^{(15)}\left(x_{N-2}\right) h^{15} \\
& +O\left(h^{16}\right), \frac{8939}{218621804544000} y^{(15)}\left(x_{0}\right) h^{15}+O\left(h^{16}\right), \ldots, \frac{127}{81729648000} y^{(15)}\left(x_{N-2}\right) \\
& \times h^{15}+O\left(h^{16}\right), \frac{20869}{82403603251200} y^{(15)}\left(x_{0}\right) h^{15}+O\left(h^{16}\right), \ldots, \\
& \frac{1}{502951680} y^{(15)}\left(x_{N-2}\right) h^{15}+O\left(h^{16}\right), \frac{551}{643778150400} y^{(15)}\left(x_{0}\right) h^{15}+O\left(h^{16}\right) \\
& \left., \ldots, \frac{1}{502951680} y^{(15)}\left(x_{N-2}\right) h^{15}+O\left(h^{16}\right)\right)^{T} .
\end{aligned}
$$

Let defined the system that approximates (1.1) as

$$
\bar{M}_{10 N \times 10 N} \overline{\bar{Y}}_{10 N}+h^{5} \bar{N}_{10 N \times(4 N+2)} \overline{\bar{F}}_{4 N+2}+\mathbf{C}_{10 N}=0
$$

where $\bar{Y}_{10 N}$ is approximated by vector $\overline{\bar{Y}}_{10 N}$,

$$
\begin{aligned}
\overline{\bar{Y}}= & \left(y_{\frac{1}{2}}, y_{1}, \ldots, y_{N-\frac{1}{2}}, y_{\frac{1}{2}}^{\prime}, \ldots, y_{N-\frac{1}{2}}^{\prime}, y_{\frac{1}{2}}^{\prime \prime}, \ldots, y_{N}^{\prime \prime}, y_{0}^{\prime \prime \prime}, y_{\frac{1}{2}}^{\prime \prime \prime},\right. \\
& \left.\ldots, y_{N}^{\prime \prime \prime}, y_{0}^{i v}, y_{\frac{1}{2}}^{i v}, \ldots, y_{N}^{i v}\right)^{T}
\end{aligned}
$$

and $\overline{\bar{F}}_{4 N+2}$ is given as $\overline{\bar{F}}_{4 N+2}=\left(f_{0}, f_{\frac{1}{2}}, \ldots, f_{N}, g_{0}, g_{\frac{1}{2}}, \ldots, g_{N}\right)^{T}$.

Subtracting equation (3.4) from (3.2) yields

$$
\bar{M}_{10 N \times 10 N} \bar{H}_{10 N}+h^{5} \bar{N}_{10 N \times(4 N+2)}(\bar{F}-\overline{\bar{F}})_{4 N+2}=\mathbb{L}(h)_{10 N},
$$

where

$$
\begin{gathered}
\bar{H}_{10 N}=\bar{Y}-\overline{\bar{Y}}=\left(e_{\frac{1}{2}}, e_{1}, \ldots, e_{N-\frac{1}{2}}, e_{\frac{1}{2}}^{\prime}, \ldots, e_{N-\frac{1}{2}}^{\prime}, e_{\frac{1}{2}}^{\prime \prime}, \ldots, e_{N}^{\prime \prime},\right. \\
\left.e_{0}^{\prime \prime \prime}, e_{\frac{1}{2}}^{\prime \prime \prime}, \ldots, e_{N}^{\prime \prime \prime}, e_{0}^{i v}, e_{\frac{1}{2}}^{i v}, \ldots, e_{N}^{i v}\right)^{T}
\end{gathered}
$$


is a vector containing the errors associated with the solution and the derivatives at the mesh points. By the Mean Value Theorem, for $i=0, \frac{1}{2}, 1, \frac{3}{2}, 2, \ldots, N$ the following identities are valid

$$
\begin{aligned}
& f\left(x_{i}, y\left(x_{i}\right), y^{\prime}\left(x_{i}\right), y^{\prime \prime}\left(x_{i}\right), y^{\prime \prime \prime}\left(x_{i}\right), y^{i v}\left(x_{i}\right)\right)-f\left(x_{i}, y_{i}, y_{i}^{\prime}, y_{i}^{\prime \prime}, y_{i}^{\prime \prime \prime}, y_{i}^{i v}\right) \\
& \quad=\left(y\left(x_{i}\right)-y_{i}\right) \frac{\partial f\left(c_{i}\right)}{\partial y}+\left(y^{\prime}\left(x_{i}\right)-y_{i}^{\prime}\right) \frac{\partial f\left(c_{i}\right)}{\partial y^{\prime}}+\left(y^{\prime \prime}\left(x_{i}\right)-y_{i}^{\prime \prime}\right) \frac{\partial f\left(c_{i}\right)}{\partial y^{\prime \prime}} \\
& \quad+\left(y^{\prime \prime \prime}\left(x_{i}\right)-y_{i}^{\prime \prime \prime}\right) \frac{\partial f\left(c_{i}\right)}{\partial y^{\prime \prime \prime}}+\left(y^{i v}\left(x_{i}\right)-y_{i}^{i v}\right) \frac{\partial f\left(c_{i}\right)}{\partial y^{i v}}, \\
& g\left(x_{i}, y\left(x_{i}\right), y^{\prime}\left(x_{i}\right), y^{\prime \prime}\left(x_{i}\right), y^{\prime \prime \prime}\left(x_{i}\right), y^{i v}\left(x_{i}\right)\right)-g\left(x_{i}, y_{i}, y_{i}^{\prime}, y_{i}^{\prime \prime}, y_{i}^{\prime \prime \prime}, y_{i}^{i v}\right) \\
& \quad=\left(y\left(x_{i}\right)-y_{i}\right) \frac{\partial g\left(\bar{c}_{i}\right)}{\partial y}+\left(y^{\prime}\left(x_{i}\right)-y_{i}^{\prime}\right) \frac{\partial g\left(\bar{c}_{i}\right)}{\partial y^{\prime}}+\left(y^{\prime \prime}\left(x_{i}\right)-y_{i}^{\prime \prime}\right) \frac{\partial g\left(\bar{c}_{i}\right)}{\partial y^{\prime \prime}} \\
& \quad+\left(y^{\prime \prime \prime}\left(x_{i}\right)-y_{i}^{\prime \prime \prime}\right) \frac{\partial g\left(\bar{c}_{i}\right)}{\partial y^{\prime \prime \prime}}+\left(y^{i v}\left(x_{i}\right)-y_{i}^{i v}\right) \frac{\partial g\left(\bar{c}_{i}\right)}{\partial y^{i v}},
\end{aligned}
$$

where $c_{i}, \bar{c}_{i}$ are intermediate points on the line segment joining $\left(x_{i}, y\left(x_{i}\right), y^{\prime}\left(x_{i}\right), y^{\prime \prime}\left(x_{i}\right), y^{\prime \prime \prime}\left(x_{i}\right), y^{i v}\left(x_{i}\right)\right)$ to $\left(x_{i}, y_{i}, y_{i}^{\prime}, y_{i}^{\prime \prime}, y_{i}^{\prime \prime \prime}, y_{i}^{i v}\right)$. Therefore,

$$
\bar{F}-\overline{\bar{F}}=\bar{J}_{(4 N+2) \times 10 N} \bar{H}_{10 N} .
$$

$\bar{J}_{(4 N+2) \times 10 N}=$

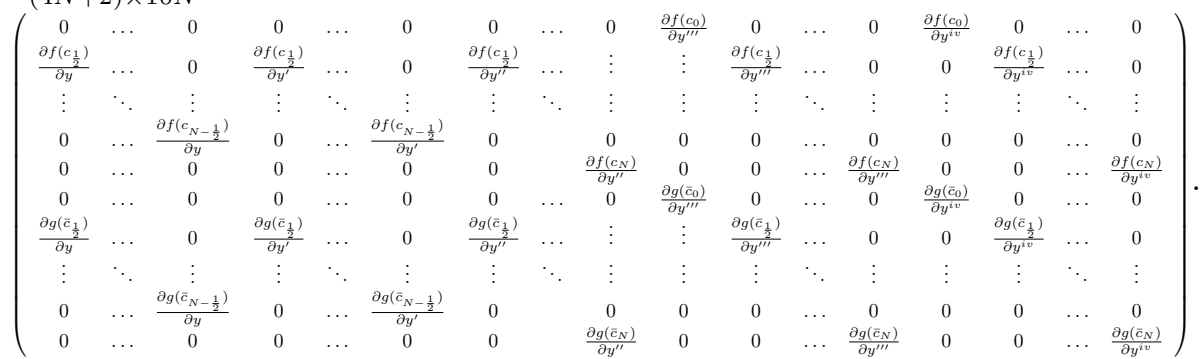

It should be noted that in view of the boundary conditions we have $e_{0}=$ $y\left(x_{0}\right)-y_{0}=0, e_{N}=y\left(x_{N}\right)-y_{N}=0, e_{0}^{\prime}=y^{\prime}\left(x_{0}\right)-y_{0}^{\prime}=0, e_{N}^{\prime}=y\left(x_{N}\right)-y_{N}=0$ and $e_{0}^{\prime \prime}=y^{\prime \prime}\left(x_{0}\right)-y_{0}^{\prime \prime}=0$. With these results, Equation (3.5) can further be written as $\bar{M}_{10 N \times 10 N} \bar{H}_{10 N}+h^{5} \bar{N}_{10 N \times(4 N+2)} \bar{J}_{(4 N+2) \times 10 N} \bar{H}_{10 N}=\mathbb{L}(h)_{10 N}$.

Let $\mathbb{M}_{10 N \times 10 N}=\left(\bar{M}_{10 N \times 10 N}+h^{5} \bar{N}_{10 N \times(4 N+2)} \bar{J}_{(4 N+2) \times 10 N}\right)$. Assuming that $\mathbb{M}$ is invertible for a sufficiently small value of $h$, therefore we can write

$$
\bar{H}_{10 N}=(\mathbb{M})_{10 N \times 10 N}^{-1} \mathbb{L}(h)_{10 N} .
$$

Consider the maximum norm $\|\bar{H}\|=\max _{i}\left|e_{i}\right|$ in $\mathbb{R}^{10 N}$ which of course will induced matrix norm in $\mathbb{R}^{10 N \times 10 N}$. By expanding $(\mathbb{M})_{10 N \times 10 N}^{-1}$ in series in powers of $h$, it is easy to see that $\left\|(\mathbb{M})_{10 N \times 10 N}^{-1}\right\|=\mathcal{O}\left(h^{-5}\right)$, which happens as a result of the growth in the uniform norm of inverse of $\mathbb{M}$ like $h^{-5}$. With this in mind and assuming that $y(x)$ has bounded derivatives in $[a, b]$ up to the necessary order, it is obvious from Equations (3.3) and (3.6) that

$$
\begin{aligned}
& \left\|\bar{H}_{10 N}\right\|=\left\|(\mathbb{M})_{10 N \times 10 N}^{-1} \mathbb{L}(h)_{10 N}\right\| \\
& \quad \leq\left\|(\mathbb{M})_{10 N \times 10 N}^{-1}\right\|\left\|\mathbb{L}(h)_{10 N}\right\|=\mathcal{O}\left(h^{-5}\right) \mathcal{O}\left(h^{15}\right)=\mathcal{O}\left(h^{10}\right) .
\end{aligned}
$$


Thus, the new method BDM5 is tenth-order convergent.

\subsection{Existence and uniqueness of the discrete solution}

The existence and uniqueness of the discrete solutions made available by the system in (3.2) is confirmed by the following result. Remember that the convergence analysis deals with the manner in which the errors grow when the step-size $h$ is relatively small. We can see that $\|\bar{H}\| \rightarrow 0$ as $h \rightarrow 0$. Hence, the following result will be achieved by assuming that $h<1$.

Theorem 1 [ see [7]]. If $f(x, \mathbb{V})$ verifies a Lipschitz condition on the variable $\mathbb{V}=\left(y, y^{\prime}, y^{\prime \prime}, y^{\prime \prime \prime}, y^{(4)}\right)$ with Lipschitz constants $L_{i}>0$ for each component, it holds that the system (3.2) has a unique solution whenever

$$
h<1 /\left(10 \mathbb{L}^{2} \bar{u}^{2}(b-a)\right)^{\frac{1}{9}},
$$

where

$$
\mathbb{L}=\max _{i=0,1,2,3,4}\left\{\mathbb{L}_{i}\right\}, \quad \bar{u}=\max _{\substack{i=1, \ldots, 10 N \\ j=1, \ldots, 4 N+2}}\left\{\left|\mathbb{U}_{i j}\right|\right\} \text { with } \mathbb{U}=\left.(\bar{M})^{-1} \bar{N}\right|_{h=1} .
$$

Proof. Consider function $\mathbb{R}^{10 N} \longrightarrow \mathbb{R}^{10 N}$ which is defined as

$$
\mathbb{W}(\mu)=\left((-\bar{M})^{-1} \mathbf{C}-h^{5}(-\bar{M})^{-1} \bar{F}(\mu)\right)^{T},
$$

where $\mu=\left(\mu_{1}, \ldots, \mu_{10 N}\right) \in \mathbb{R}^{10 N}$ and $\bar{F}(\mu)$ denotes the vector upon substitut$\operatorname{ing} \bar{Y} \rightarrow \mu$ in the terms $f_{j}, f_{j}^{\prime}, f_{j}^{\prime \prime}, f_{j}^{\prime \prime \prime}$ and $f_{j}^{(4)}$. By setting $\mu=\bar{Y}$, the system in (3.2) takes the form $\mu=\mathbb{W}(\mu)$. Hence, the existence and uniqueness of solution of the systems in (3.2) is the same as $\mu=\mathbb{W}(\mu)$. Consider the maximum norm

$$
\|\mu\|=\max _{1 \leq i \leq 10 N}\left\{\left|\mu_{i}\right|\right\} \in \mathbb{R}^{10 N}
$$

We have

$$
\begin{aligned}
\left|(\mathbb{W}(\mu))_{i}-\left(\mathbb{W}\left(\mu^{*}\right)\right)_{i}\right| & =\left|h^{5}\left[\bar{M}^{-1} \bar{N}\left(\bar{F}(\mu)-\bar{F}\left(\mu^{*}\right)\right)\right]_{i}\right| \\
& \leq h^{5} \bar{u} \sum_{j=0}^{10 N} \mathbb{L}\left|\mu_{j}-\mu_{j}^{*}\right|
\end{aligned}
$$

where

$$
\mathbb{L}=\max _{i=0,1,2,3,4}\left\{\mathbb{L}_{i}\right\}, \quad \bar{u}=\max _{\substack{i=1, \ldots, 10 N \\ j=1, \ldots, 4 N+2}}\left\{\left|\mathbb{U}_{i j}\right|\right\}
$$

and $\mathbb{U}_{i j}$ the entries of matrix $\mathbb{U}=\left.(\bar{M})^{-1} \bar{N}\right|_{h=1}$. Applying the Cauchy-Schwartz inequality to (3.7) yields

$$
\begin{aligned}
& \left|\mathbb{W}(\mu)-\mathbb{W}\left(\mu^{*}\right)\right|=\max _{1 \leq i \leq 10 N}\left\{\left|(\mathbb{W}(\mu))_{i}-\left(\mathbb{W}\left(\mu^{*}\right)\right)_{i}\right|\right\} \\
& \quad \leq h^{5} \bar{u} \mathbb{L} \sqrt{(10 N)}\left\|\mu-\mu^{*}\right\|=h^{5} \bar{u} \mathbb{L} \sqrt{\left(10\left(\frac{b-a}{h}\right)\right)}\left\|\mu-\mu^{*}\right\|=\bar{k}\left\|\mu-\mu^{*}\right\|
\end{aligned}
$$

with $\bar{k}=h^{5} \bar{u} \mathbb{L} \sqrt{(10(b-a) / h)}$. As long as $\bar{k}<1, \mathbb{W}$ will become a contraction map, which end the proof according to the Banach's Fixed-Point Theorem. 


\section{Implementation}

The systems in (3.2) is solved by adopting the Newton's iteration method enhanced by the FindRoot[] command in Wolfram Mathematica. For the method to show good convergence, the initial guess has to be chosen close enough to the root. To achieved this, averaged values of the given variables are considered as the initial guesses. These are obtained as

$$
\begin{aligned}
y_{\frac{j}{2}} & =\frac{\left(y(a)-j \frac{h}{2} y^{\prime}(a)-j(j-j) y^{\prime \prime}(a)\right)+\left(y(b)-(4 N-j) \frac{h}{2} y^{\prime}(b)-(4 N-j) \frac{h}{2} y^{\prime \prime}(b)\right)}{2}, \\
j=1, \ldots,(4 N-1), & \\
y_{\frac{j}{2}}^{\prime} & =\frac{y^{\prime}(a)+y^{\prime}(b)}{2}, \quad y_{\frac{j}{2}}^{\prime \prime}=\frac{y^{\prime}(b)-y^{\prime}(a)}{N h}, \quad j=1, \ldots,(4 N-1), \\
y_{\frac{j}{2}}^{\prime \prime \prime} & =\frac{y^{\prime \prime}(a)}{N h}, \quad y_{\frac{j}{2}}^{(4)}=1, \quad j=0, \ldots, 4 N .
\end{aligned}
$$

The following algorithm explains the computational procedure for the implementation of the proposed method.

\section{ALGORITHM}

Input:

Integration interval $\rightarrow[a, b]$

Number of steps $\rightarrow N$

Boundary values, $\rightarrow y_{a}, y_{a}^{\prime}, y_{a}^{\prime \prime}, y_{b}, y_{b}^{\prime}$

Output: sol $\rightarrow$ Discrete approximate solution of the BVP (1.1).

1. Define the following Let $n=0,2, \ldots,(N-2), x_{0}=a, x_{N}=b$, $h=\frac{b-a}{N}, y_{0}=y_{a}, y_{0}^{\prime}=y_{a}^{\prime}, y_{0}^{\prime \prime}=y_{a}^{\prime \prime}$, $y_{N}=y_{b}, y_{N}^{\prime}=y_{b}^{\prime}$.

2. Solve solve for $\bar{Y}$ with the method whose formulas are reported in Table 1.

3. Discrete solution Obtain sol $=\left\{\left(x_{i}, y_{i}\right)\right\}_{i=0,1, \ldots, N}$.

4. End

\section{$5 \quad$ Numerical examples}

In this section five numerical examples are presented to demonstrate the performance of the derived method, BDM5. In each of the examples considered, the absolute errors are obtained as $\operatorname{err}(n)=\left|y\left(x_{n}\right)-y_{n}\right|$. The computational time in seconds used by BDM5 denoted as CPU are measured, NA denotes "data Not Available". The computational work has been done on a personal computer with configuration i7-7500U, 2.70 GHz using double precision arithmetic.

Example 1. The boundary-value problem

$$
\begin{aligned}
& y^{(5)}(x)-y(x)=-(15+10 x) e^{x}, \quad 0 \leq x \leq 1, \\
& y(0)=y(1)=y^{\prime \prime}(0)=0, y^{\prime}(0)=1, y^{\prime}(1)=-e,
\end{aligned}
$$

whose theoretical solution is $y(x)=x(x-1) e^{x}$ is considered as our first test problem. This particular problem has appeared in virtually all literature that 
Table 2. Comparison of observed maximum absolute errors on Example 1 with $h=1 / 10$.

\begin{tabular}{lllll}
\hline$x$ & y-computed & y-exact & MAE in [12] & MAE in BDM5 \\
\hline 0 & 0 & 0 & & 0 \\
0.1 & 0.09946538262680828 & 0.0994653826268083 & $2.17 \times 10^{-11}$ & $1.978 \times 10^{-22}$ \\
0.2 & 0.19542444130562717 & 0.1954244413056272 & $6.87 \times 10^{-11}$ & $1.285 \times 10^{-21}$ \\
0.3 & 0.28347034959096065 & 0.2834703495909607 & $1.19 \times 10^{-10}$ & $3.389 \times 10^{-21}$ \\
0.4 & 0.35803792743390487 & 0.3580379274339049 & $1.56 \times 10^{-10}$ & $6.017 \times 10^{-21}$ \\
0.5 & 0.41218031767503204 & 0.4121803176750321 & $1.71 \times 10^{-10}$ & $8.322 \times 10^{-21}$ \\
0.6 & 0.43730851209372215 & 0.4373085120937221 & $1.59 \times 10^{-10}$ & $9.386 \times 10^{-21}$ \\
0.7 & 0.42288806856880007 & 0.4228880685688001 & $1.22 \times 10^{-10}$ & $8.553 \times 10^{-21}$ \\
0.8 & 0.35608654855879481 & 0.3560865485587949 & $7.15 \times 10^{-11}$ & $5.791 \times 10^{-21}$ \\
0.9. & 0.22136428000412547 & 0.2213642800041255 & $2.25 \times 10^{-11}$ & $2.106 \times 10^{-21}$ \\
1.0 & 0 & 0 & 0 & 0 \\
\hline CUP & & & NA & 0.234375 \\
\hline
\end{tabular}

are available on fifth-order BVPs (see, $[3,5,12,15,16,17,18,19])$. The exact, computed solution and absolute errors given by the proposed method are shown in Table 2 with the absolute error obtained with the spline-based method in [12]. Table 3 shows the maximum absolute error (MAE) for different mesh points provided by BDM5 and the best method in [12], while the absolute errors of the derived method presented in 2 are further compared with some methods in [8] in Table 5.

Table 3. Comparison of maximum absolute errors (MAE) on Example 1.

\begin{tabular}{llll}
\hline Method used $/ h$ & $\frac{1}{10}$ & $\frac{1}{20}$ & $\frac{1}{40}$ \\
\hline$[12]$ & $1.71 \times 10^{-10}$ & $1.86 \times 10^{-13}$ & $1.61 \times 10^{-13}$ \\
BDM5 & $9.39 \times 10^{-21}$ & $9.14 \times 10^{-24}$ & $8.92 \times 10^{-27}$ \\
\hline
\end{tabular}

Table 4. Maximum absolute errors (MAE) and rate of convergence (ROC) obtained for Example 1.

\begin{tabular}{llllll}
\hline$N$ & 4 & 8 & 16 & 32 & 64 \\
\hline$M A E$ & $8.050 \times 10^{-17}$ & $8.746 \times 10^{-20}$ & $8.504 \times 10^{-23}$ & $8.297 \times 10^{-26}$ & $8.121 \times 10^{-29}$ \\
$R O C$ & - & 9.85 & 10.01 & 10.00 & 9.996 \\
\hline$N$ & 128 & & & \\
\hline$M A E$ & $8.00 \times 10^{-32}$ & & & & \\
$R O C$ & 9.987 & & & \\
\hline
\end{tabular}

In order to determined whether the performance of the proposed method corresponds to the theoretical order of convergence of the method, the approximate rate of convergence (ROC) of the proposed method was calculated on 
Table 5. Comparison of errors obtained with BDM5 and some methods in [8] on Example 1 with $h=1 / 10$.

\begin{tabular}{llllllll}
\hline$x$ & BDM5 & HPM & B-spline & VIM & ADM & ITM & VIMHP \\
\hline 0 & 0 & 0 & 0 & 0 & 0 & 0 & 0 \\
0.1 & $1.98 e-22$ & $3.0 e-11$ & $8.0 \times 10^{-3}$ & $3.0 \times 10^{-11}$ & $3.0 \times 10^{-11}$ & $3.0 \times 10^{-11}$ & $3.0 \times 10^{-11}$ \\
0.2 & $1.29 e-21$ & $2.0 e-10$ & $1.2 \times 10^{-3}$ & $2.0 \times 10^{-10}$ & $2.0 \times 10^{-10}$ & $2.0 \times 10^{-10}$ & $2.0 \times 10^{-10}$ \\
0.3 & $3.39 e-21$ & $4.0 e-10$ & $5.0 \times 10^{-3}$ & $4.0 \times 10^{-10}$ & $4.0 \times 10^{-10}$ & $4.0 \times 10^{-10}$ & $4.0 \times 10^{-10}$ \\
0.4 & $6.02 e-21$ & $8.0 e-10$ & $3.0 \times 10^{-3}$ & $8.0 \times 10^{-10}$ & $8.0 \times 10^{-10}$ & $8.0 \times 10^{-10}$ & $8.0 \times 10^{-10}$ \\
0.5 & $8.32 e-21$ & $1.2 e-9$ & $8.0 \times 10^{-3}$ & $1.2 \times 10^{-9}$ & $1.2 \times 10^{-9}$ & $1.2 \times 10^{-9}$ & $1.2 \times 10^{-9}$ \\
0.6 & $9.39 e-21$ & $2.0 e-9$ & $6.0 \times 10^{-3}$ & $2.0 \times 10^{-9}$ & $2.0 \times 10^{-9}$ & $2.0 \times 10^{-9}$ & $2.0 \times 10^{-9}$ \\
0.7 & $8.55 e-21$ & $2.2 e-9$ & $0.0 \times 10^{-3}$ & $2.2 \times 10^{-9}$ & $2.2 \times 10^{-9}$ & $2.2 \times 10^{-9}$ & $2.2 \times 10^{-9}$ \\
0.8 & $5.79 e-21$ & $1.9 e-9$ & $9.0 \times 10^{-3}$ & $1.9 \times 10^{-9}$ & $1.9 \times 10^{-9}$ & $1.9 \times 10^{-9}$ & $1.9 \times 10^{-9}$ \\
0.9 & $2.11 e-21$ & $1.4 e-9$ & $9.0 \times 10^{-3}$ & $1.4 \times 10^{-9}$ & $1.4 \times 10^{-9}$ & $1.4 \times 10^{-9}$ & $1.4 \times 10^{-9}$ \\
1.0 & 0 & 0 & 0 & 0 & 0 & 0 & 0 \\
\hline CUP & 0.234375 & NA & NA & NA & NA & NA & NA \\
\hline
\end{tabular}

Example 1 using the relation

$$
R O C=\log _{2}\left(M A E_{2 h} / M A E_{h}\right),
$$

where $M A E_{2 h}$ and $M A E_{h}$ denote maximum absolute error on the grid with mesh sizes $2 h$ and $h$ respectively. See Table 4 for the results.

Example 2. Another test problem considered in this paper is the BVP

$$
\begin{aligned}
& y^{(5)}(x)+\sin x y(x)=\cos x(1+\sin x)+\sin x(\sin x-1), \quad 0 \leq x \leq 1, \\
& y(0)=1, \quad y(1)=\cos (1)+\sin (1), \\
& y^{\prime}(0)=1, \quad y^{\prime}(1)=\cos (1)-\sin (1), \quad y^{\prime \prime}(0)=-1,
\end{aligned}
$$

for which the analytical solution is $y(x)=\cos (x)+\sin (x),([16])$. The solution curve and the discrete approximations obtained with the proposed method, together with the absolute errors for $h=1 / 20$ are shown in Figure 1 . The comparison of the maximum absolute errors obtained with BDM5 and those obtained with the non-polynomial spline method in [16] are presented in Table 6 .

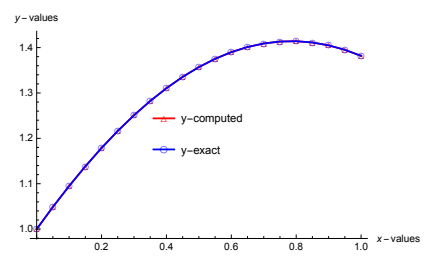

(a)

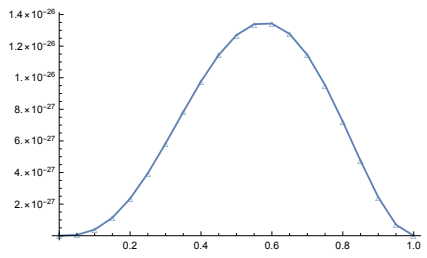

(b)

Figure 1. (a) and (b) show the solution curve and absolute errors for Example 5 taking $h=1 / 20$ using BDM5. 
Table 6. Comparison of maximum absolute errors (MAE) on Example 2.

\begin{tabular}{lll}
\hline Method used $/ h$ & $1 / 10$ & $1 / 20$ \\
\hline$[16]$ & $3.1495 \times 10^{-12}$ & $2.17160 \times 10^{-13}$ \\
BDM5 & $1.3697 \times 10^{-23}$ & $1.34293 \times 10^{-26}$ \\
\hline
\end{tabular}

Example 3. The next test problem considered in this paper is the BVP

$$
\begin{aligned}
& y^{(5)}(x)+y(x)=4 e^{x} \cos x+2 e^{x}(1-\sin x)+5 e^{x} \sin x, \quad 0 \leq x \leq 1, \\
& y(0)=1, \quad y(1)=e(1-\sin (1)), \\
& y^{\prime}(0)=0, \quad y^{\prime}(1)=e(\cos (1)+\sin (1)-1), y^{\prime \prime}(0)=-1,
\end{aligned}
$$

for which the exact solution is $y(x)=e^{x}(1-\sin (x))$. The problem was also solved by the method in [16]. Table 7 shows the observed absolute errors with BDM5 and that of the non-polynomial spline method in [16]. Table 8 shows the maximum absolute errors of the methods at different mesh points.

Table 7. Comparison of observed absolute errors on Example 3 with $h=1 / 10$.

\begin{tabular}{lllll}
\hline$x$ & y-computed & y-exact & MAE in BDM5 & MAE in [16] \\
\hline 0 & 1 & 1. & 0 & 0 \\
0.1 & 0.994837929345443913 & $0.9948379293454439^{`}$ & $6.6416 \times 10^{-23}$ & $3.5971 \times 10^{-11}$ \\
0.2 & 0.978747489565246898 & $0.9787474895652469^{\natural}$ & $4.1769 \times 10^{-22}$ & $1.1782 \times 10^{-10}$ \\
0.3 & 0.950948253797513255 & $0.9509482537975132^{`}$ & $1.0580 \times 10^{-21}$ & $2.0565 \times 10^{-10}$ \\
0.4 & 0.910880796870703093 & $0.910880796870703^{6}$ & $1.7971 \times 10^{-21}$ & $2.7051 \times 10^{-10}$ \\
0.5 & 0.858282187486513235 & $0.8582821874865133^{6}$ & $2.3684 \times 10^{-21}$ & $2.9465 \times 10^{-10}$ \\
0.6 & 0.793273134118417270 & $0.7932731341184172^{6}$ & $2.5345 \times 10^{-21}$ & $2.7173 \times 10^{-10}$ \\
0.7 & 0.716457595595207513 & $0.7164575955952076^{6}$ & $2.1813 \times 10^{-21}$ & $2.0715 \times 10^{-10}$ \\
0.8 & 0.629035587892216727 & $0.6290355878922167^{`}$ & $1.3870 \times 10^{-21}$ & $1.1832 \times 10^{-10}$ \\
0.9. & 0.532929807184232775 & $0.5329298071842328^{6}$ & $4.7062 \times 10^{-22}$ & $3.5059 \times 10^{-11}$ \\
1.0 & $e(1-\sin (1))$ & $0.4309265412802028^{6}$ & 0 & 0 \\
\hline CUP & & & NA & 0.25 \\
\hline
\end{tabular}

Table 8. Comparison of maximum absolute errors (MAE) on Example 3.

\begin{tabular}{lll}
\hline Method used $/ h$ & $1 / 10$ & $1 / 20$ \\
\hline$[16]$ & $1.02830 \times 10^{-10}$ & $1.10520 \times 10^{-12}$ \\
BDM5 & $2.53445 \times 10^{-21}$ & $2.50707 \times 10^{-24}$ \\
\hline
\end{tabular}

Example 4. The boundary value problem

$$
\begin{aligned}
& y^{(5)}(x)=e^{-x} y^{2}(x), \quad 0 \leq x \leq 1, \\
& y(0)=1, \quad y(1)=e, \quad y^{\prime}(0)=1, \quad y^{\prime}(1)=e, \quad y^{\prime \prime}(0)=1,
\end{aligned}
$$

for which the exact solution is $y(x)=e^{x}$ is also considered. This problem has also been considered in the works by $[8,15,18,19]$. Table 9 displays the observed 
absolute errors obtained with BDM5 and those of the methods appeared in [8]. In particular, the errors in the last column in Table 9 are those generated by the variational iterative method using He's polynomial (VIMHP) of degree fourteen.

Table 9. Comparison of observed absolute errors on Example 4 using BDM5 and the methods in [8] with $h=1 / 10$.

\begin{tabular}{llllllll}
\hline$x$ & BDM5 & HPM & B-spline & VIM & ADM & ITM & VIMHP \\
\hline 0 & 0 & 0 & 0 & 0 & 0 & 0 & 0 \\
0.1 & $9.6 \times 10^{-25}$ & $1.0 \times 10^{-9}$ & $-7.0 \times 10^{-4}$ & $1.0 \times 10^{-9}$ & $1.0 \times 10^{-9}$ & $1.0 \times 10^{-9}$ & $1.0 \times 10^{-9}$ \\
0.2 & $6.2 \times 10^{-24}$ & $2.0 \times 10^{-9}$ & $-7.2 \times 10^{-4}$ & $2.0 \times 10^{-9}$ & $2.0 \times 10^{-9}$ & $2.0 \times 10^{-9}$ & $2.0 \times 10^{-9}$ \\
0.3 & $1.6 \times 10^{-23}$ & $1.0 \times 10^{-8}$ & $4.1 \times 10^{-4}$ & $1.0 \times 10^{-8}$ & $1.0 \times 10^{-8}$ & $1.0 \times 10^{-8}$ & $1.0 \times 10^{-8}$ \\
0.4 & $2.9 \times 10^{-23}$ & $2.0 \times 10^{-8}$ & $4.6 \times 10^{-4}$ & $2.0 \times 10^{-8}$ & $2.0 \times 10^{-8}$ & $2.0 \times 10^{-8}$ & $2.0 \times 10^{-8}$ \\
0.5 & $4.0 \times 10^{-23}$ & $3.1 \times 10^{-8}$ & $4.7 \times 10^{-4}$ & $3.1 \times 10^{-8}$ & $3.1 \times 10^{-8}$ & $3.1 \times 10^{-8}$ & $3.1 \times 10^{-8}$ \\
0.6 & $4.5 \times 10^{-23}$ & $3.7 \times 10^{-8}$ & $4.8 \times 10^{-4}$ & $3.7 \times 10^{-8}$ & $3.7 \times 10^{-8}$ & $3.7 \times 10^{-8}$ & $3.7 \times 10^{-8}$ \\
0.7 & $4.1 \times 10^{-23}$ & $4.1 \times 10^{-8}$ & $3.9 \times 10^{-4}$ & $4.1 \times 10^{-8}$ & $4.1 \times 10^{-8}$ & $4.1 \times 10^{-8}$ & $4.1 \times 10^{-8}$ \\
0.8 & $2.8 \times 10^{-23}$ & $3.1 \times 10^{-8}$ & $3.1 \times 10^{-4}$ & $3.1 \times 10^{-8}$ & $3.1 \times 10^{-8}$ & $3.1 \times 10^{-8}$ & $3.1 \times 10^{-8}$ \\
0.9 & $1.0 \times 10^{-23}$ & $1.4 \times 10^{-8}$ & $1.6 \times 10^{-4}$ & $1.4 \times 10^{-8}$ & $1.4 \times 10^{-8}$ & $1.4 \times 10^{-8}$ & $1.4 \times 10^{-8}$ \\
1.0 & 0 & 0 & 0 & 0 & 0 & 0 & 0 \\
\hline CUP & 0.203125 & NA & NA & NA & NA & NA & NA \\
\hline
\end{tabular}

Table 10. Observed MAE of BDM5 and [15] and ROC of BDM5 for Example 5.

\begin{tabular}{llll}
\hline$h$ & MAE in $[15]$ & MAE in BDM5 & ROC (BDM5) \\
\hline$\frac{1}{8}$ & $5.06 \times 10^{-4}$ & $5.684 \times 10^{-16}$ & - \\
$\frac{1}{16}$ & $3.04 \times 10^{-5}$ & $5.741 \times 10^{-19}$ & 9.9514 \\
$\frac{1}{32}$ & $2.42 \times 10^{-6}$ & $5.635 \times 10^{-22}$ & 9.9927 \\
$\frac{1}{64}$ & $6.75 \times 10^{-7}$ & $5.508 \times 10^{-25}$ & 9.9986 \\
$\frac{1}{128}$ & $1.71 \times 10^{-7}$ & $5.380 \times 10^{-28}$ & 9.9997 \\
$\frac{1}{256}$ & $4.32 \times 10^{-8}$ & $5.000 \times 10^{-31}$ & 10.0725 \\
\hline
\end{tabular}

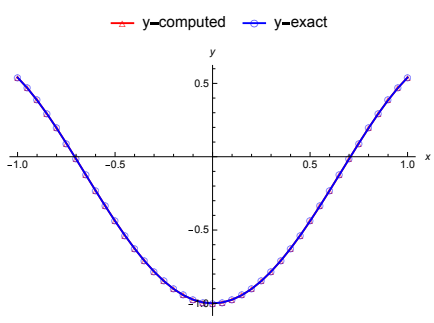

(a)

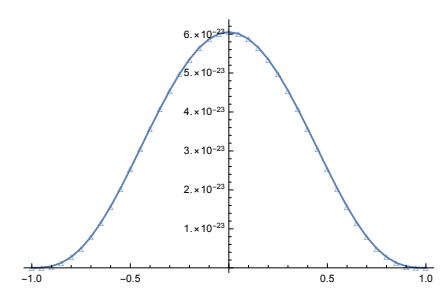

(b)

Figure 2. (a) and (b) show the solution curve and absolute errors for Example 5 taking $h=\frac{1}{20}$ using BDM5. 
Example 5. We also considered the BVP

$$
\begin{aligned}
& y^{(5)}(x)=19 x \cos x+2 x^{3} \cos x+41 \sin x-2 x^{2} \sin x-x y(x), \quad 0 \leq x \leq 1, \\
& y(-1)=1, y(1)=\cos (1), \\
& y^{\prime}(-1)=-y^{\prime}(1)=-4 \cos (1)+\sin (1), \\
& y^{\prime \prime}(-1)=3 \cos (1)-8 \sin (1),
\end{aligned}
$$

for which the exact solution is $y(x)=\left(2 x^{2}-1\right) \cos (x)$. This problem also appeared in [15]. The solution curve and the approximate solution provided by the proposed method taking $h=1 / 20$ and the absolute errors presented in Figure 2. Table 10 displays the maximum absolute error generated by BDM5 and the sextic spline solution in [15].

\section{Conclusions}

A method of tenth order has been derived and analysed in this work for the direct approximation of fifth-order boundary value problems in ordinary differential equations. The derivation of the method is simple and flexible in application to solve a variety of boundary value problems with different boundary conditions. The theoretical analysis of the method confirmed the tenth-order convergence while its application to some numerical examples established the efficiency and high accuracy of the proposed method which make it competitive with other methods in the literature.

\section{References}

[1] R.P. Agarwal. Boundary Value Problems from Higher Order Differential Equations. World Scientific, 1986. https://doi.org/10.1142/0266.

[2] G. Akram. Solution of the system of fifth order boundary value problem using sextic spline. Journal of the Egyptian Mathematical Society, 23(2):406-409, 2015. https://doi.org/10.1016/j.joems.2014.04.009.

[3] H.N. Çaglar, S.H. Çaglar and E.H. Twizell. The numerical solution of fifthorder boundary value problems with sixth-degree B-spline functions. $A p$ plied Mathematics Letters, 12(5):25-30, 1999. https://doi.org/10.1016/s08939659(99)00052-x.

[4] J.D. Lambert. Computational Methods in Ordinary Differential Equations. Wiley and Son Ltd, 1973.

[5] X. Lv and M. Cui. An efficient computational method for linear fifth-order twopoint boundary value problems. Journal of Computational and Applied Mathematics, 234(5):1551-1558, 2010. https://doi.org/10.1016/j.cam.2010.02.036.

[6] M.I. Modebei, R.B. Adeniyi, S.N. Jator and H. Ramos. A block hybrid integrator for numerically solving fourth-order initial value problems. $\quad$ Applied Mathematics and Computation, 346:680-694, 2019. https://doi.org/10.1016/j.amc.2018.10.080.

[7] M.I. Modebei, S.N. Jator and H. Ramos. Block hybrid method for the numerical solution of fourth order boundary value problems. Journal of Computational and Applied Mathematics, 377:112876, 2020. https://doi.org/10.1016/j.cam.2020.112876. 
[8] M.A. Noor and S.T. Mohyud-Din. Variational iteration method for fifth-order boundary value problems using Hes polynomials. Mathematical Problems in Engineering, 2008, 2008. https://doi.org/10.1155/2008/954794.

[9] B.T. Olabode and A.L. Momoh. Continuous hybrid multistep methods with legendre basis function for direct treatment of second order stiff ODEs. America Journal of Computational and Applied Mathematics, 6(2):38-49, 2016. https://doi.org/10.5923/j.ajcam.20160602.03.

[10] H. Ramos. Development of a new Runge-Kutta method and its economical implementation. Computational and Mathematical Methods, 1(2):e1016, 2019. https://doi.org/10.1002/cmm4.1016.

[11] H. Ramos and M.A. Rufai. A third-derivative two-step block Falknertype method for solving general second-order boundary-value systems. Mathematics and Computers in Simulation, 165:139-155, 2019. https://doi.org/10.1016/j.matcom.2019.03.003.

[12] J. Rashidinia, R. Jalilian and K. Farajeyan. Spline approximate solution of fifth-order boundary-value problem. Applied Mathematics and Computation, 192(1):107-112, 2007. https://doi.org/10.1016/j.amc.2007.02.124.

[13] S.M. Redd. Collocation method for fifth order boundary value problems by using Quintic B-splines. International Journal Of Engineering And Computer Science, 5(8), 2016. https://doi.org/10.18535/ijecs/v5i8.48.

[14] M. Sadaf and G. Akram. A Legendre-homotopy method for the solutions of higher order boundary value problems. Journal of King Saud University - Science, 32(1):537-543, 2020. https://doi.org/10.1016/j.jksus.2018.08.002.

[15] S.S. Siddiqi and G. Akram. Sextic spline solutions of fifth order boundary value problems. Applied Mathematics Letters, 20(5):591-597, 2007. https://doi.org/10.1016/j.aml.2006.06.012.

[16] S.S. Siddiqi and M. Sadaf. Application of non-polynomial spline to the solution of fifth-order boundary value problems in induction motor. Journal of the Egyptian Mathematical Society, 23(1):20-26, 2015. https://doi.org/10.1016/j.joems.2014.01.003.

[17] K.N.S. Kasi Viswanadham and S. Ballem. Numerical solution of fifth order boundary value problems by Galerkin method with quartic Bsplines. International Journal of Computer Applications, 77(17):7-12, 2013. https://doi.org/10.5120/13613-1382.

[18] A.-M. Wazwaz. The numerical solution of fifth-order boundary value problems by the decomposition method. Journal of Computational and Applied Mathematics, 136(1):259-270, 2001. https://doi.org/10.1016/s0377-0427(00)00618-x.

[19] J. Zhang. The numerical solution of fifth-order boundary value problems by the variational iteration method. Computers 85 Mathematics with Applications, 58(11):2347-2350, 2009. https://doi.org/10.1016/j.camwa.2009.03.073. 